\title{
Several properties on quasi-class $A$ operators
}

\author{
M.H.M. Rashid
}

\begin{abstract}
In this paper, we shall show a similar results corresponding the results of M. Ito [6] for quasi-class $A$ introduced in [7] as a class of operators including class $A$ and $p$-quasihyponormal. Moreover, we shall show several properties on quasi-class $A$ which corresponding to the properties on class $A$ and $p$-quasihyponormal.
\end{abstract}

\section{Introduction}

Let $\mathcal{H}$ be a complex Hilbert space, and let $\mathbf{B}(\mathcal{H})$ denote the algebra of all bounded linear operators on $\mathcal{H}$. If $T \in \mathbf{B}(\mathcal{H})$, we shall write $\operatorname{ker}(T), \operatorname{ran}(T)$ for the null space and range of $T$, respectively. An operator $T$ is said to be positive (denoted by $T \geq 0$ ) if $\langle T x, x\rangle \geq 0$ for all $x \in \mathcal{H}$ and also $T$ is said to be strictly positive (denoted by $T>0$ ) if $T$ is positive and invertible.

Recall $([1,8,9])$ that an operator $T$ is called $p$-quasihyponormal if $T^{*}\left(\left(T^{*} T\right)^{p}-\right.$ $\left.\left(T T^{*}\right)^{p} T\right) \geq 0$ for $p \in(0,1]$, and $T$ is called paranormal if $\left\|T^{2} x\right\| \geq\|T x\|^{2}$ for all unit vector $x \in \mathcal{H}$. Following $[5,6,10]$ we say that $T \in \mathbf{B}(\mathcal{H})$ belongs to class $A$ if $\left|T^{2}\right| \geq|T|^{2}$ and $T$ is called normaloid if $\left\|T^{n}\right\|=\|T\|^{n}$, for $n \in \mathbb{N}$ (equivalently, $\|T\|=r(T)$, the spectral radius of $T$ ). Recall [2], an operator $T \in \mathbf{B}(\mathcal{H})$ is said to be $w$-hyponormal if $|\widetilde{T}| \geq|T| \geq\left|\widetilde{T^{*}}\right|$. We remark that $w$-hyponormal operator is defined by using Aluthge transformation $\widetilde{T}=|T|^{\frac{1}{2}} U|T|^{\frac{1}{2}}$. An operator $T$ is said to be quasi-class $A$ if

$$
T^{*}\left|T^{2}\right| T \geq T^{*}|T|^{2} T \text {. }
$$

\footnotetext{
Key Words: Quasi-class $A$ operators, $p$-quasihyponormal operators, Class $A$ operators. 2010 Mathematics Subject Classification: Primary 47A53, 47A55; Secondary 47A10, 47A11, 47A20.

Received: December, 2010.

Accepted: February, 2012.
} 
The quasi-class $A$ operators were introduced, and their properties were studied in [7]. (see also [4] ). In particular, it was shown in [7] that the class of quasi-class $A$ operators contains properly classes of class $A$ and $p$ quasihyponormal operators.

Quasi-class A operators were independently introduced by Jeon and Kim [7]. They gave an example of a quasi-class $A$ operator which is not paranormal nor normaloid. Jeon and Kim example show that neither the class paranormal operators nor the class of quasi-class $A$ contains the other. we shall denote classes of $p$-quasihyponormal operators, paranormal operators, normaloid operators, class $A$ operators, and quasi-class $A$ operators by $2 \mathcal{H}(p), \mathcal{P N}, \mathcal{N}, \mathcal{A}$, and $\mathcal{L A}$, respectively. It is well known that

$$
\mathcal{A} \subset \mathcal{P \mathcal { N }} \subset \mathcal{N} \quad \text { and } \quad \mathcal{Q H}(p) \subset \mathcal{P \mathcal { N }} \subset \mathcal{N},
$$

also, the following inclusions holds;

$$
\mathcal{A} \subset \mathcal{Q} \mathcal{A} \quad \text { and } \quad 2 \mathcal{H}(p) \subset \mathcal{Q} \mathcal{A}
$$

Recently, M. Ito [6] showed the following results on powers of class $A$ operators.

Theorem 1.1. Let $T$ be an invertible and class $A$ operator. Then the following assertions holds;

1. $\left|T^{n}\right|^{\frac{1}{2 n}} \geq\left(T^{*}\left|T^{n-1}\right|^{\frac{2}{n-1}} T\right)^{\frac{1}{2}} \geq|T|^{2}$ for $n=2,3, \cdots$.

2. $\left|T^{n+1}\right|^{\frac{2 n}{n+1}} \geq\left|T^{n}\right|^{2}$ for all positive integer $n$.

3. $\left|T^{2 n}\right| \geq\left|T^{n}\right|^{2}$ for all positive integer $n$.

4. $|T|^{2} \leq\left|T^{2}\right| \leq \ldots \leq\left|T^{n}\right|^{\frac{2}{n}}$ for all positive integer $n$.

5. $\left|T^{-2}\right| \geq\left|T^{-1}\right|^{2}$.

Theorem 1.2. Let $T$ be an invertible and class $A$. Then the following assertions holds;

1. $\left|T^{*}\right|^{2} \geq\left(T\left|T^{(n-1) *}\right| \frac{2}{n-1} T^{*}\right)^{\frac{1}{2}} \geq\left|T^{* n}\right|^{\frac{2}{n}}$ for $n=2,3, \cdots$.

2. $\left|T^{n *}\right|^{2} \geq \mid T^{\left.(n+1) *\right|^{\frac{2 n}{n+1}}}$ for all integer $n=2,3, \cdots$.

3. $\left|T^{n *}\right|^{2} \geq\left|T^{2 n *}\right|$ for all integer $n=2,3, \cdots$. 
4. $\left|T^{*}\right|^{2} \geq\left|T^{2 *}\right| \geq \cdots \geq\left|T^{n *}\right|^{\frac{2}{n}}$.

In this paper, we shall show similar results corresponding to Theorem 1.1 and Theorem 1.2 for a quasi-class $A$ operators. Moreover, we shall show several properties on quasi-class $A$ operators.

\section{Results}

We begin this section by introducing the following famous inequality which is quite useful for the study of quasi-class $A$ operators.

Theorem 2.1. (Löwner-Heinz Theorem) If $A \geq B \geq 0$, then $A^{\alpha} \geq B^{\alpha}$ for any $\alpha \in[0,1]$.

Theorem 2.2. Let $T$ be an invertible operator such that

$$
\left(T^{*}\left|T^{n-1}\right|^{2 k} T\right)^{\frac{1}{(n-1) k+1}} \geq|T|^{2}
$$

for some $k>0$ and $n=2,3, \cdots$. Then for any fixed $\delta \geq-1$,

$$
f_{n, \delta}(\ell)=T^{*^{n-1}}\left(T^{*}\left|T^{n-1}\right|^{2 \ell} T\right)^{\frac{\delta+1}{(n-1) \ell+1}} T^{n-1}
$$

is increasing for $\ell \geq \max \left\{k, \frac{\delta}{n-1}\right\}$.

We need the following Lemma in order to give a proof of Theorem 2.2.

Lemma 2.3. [6, Theorem $C]$ Let $A$ and $B$ be positive invertible operators such that

$$
\left(B^{\frac{1}{2}} A B^{\frac{1}{2}}\right)^{\frac{\beta_{0}}{\alpha_{0}+\beta_{0}}} \geq B
$$

holds for fixed $\alpha_{0} \geq 0$ and $\beta_{0} \geq 0$ with $\alpha_{0}+\beta_{0}>0$. Then for any fixed $\delta \geq-\beta_{0}$,

$$
g(\lambda, \mu)=B^{\frac{-\mu}{2}}\left(B^{\frac{\mu}{2}} A^{\lambda} B^{\frac{\mu}{2}}\right)^{\frac{\delta+\beta_{0} \mu}{\alpha_{0} \lambda+\beta_{0} \mu}} B^{\frac{-\mu}{2}}
$$

is an increasing function of both $\lambda$ and $\mu$ for $\lambda \geq 1$ and $\mu \geq 1$ such that $\alpha_{0} \lambda \geq \delta$.

Proof of Theorem 2.2. Let $T=U|T|$ be the polar decomposition of $T$. We remark that $U$ is unitary since $T$ is invertible. Suppose that

$$
\left(T^{*}\left|T^{n-1}\right|^{2 k} T\right)^{\frac{1}{(n-1) k+1}} \geq|T|^{2} .
$$


Since

$$
\begin{aligned}
\left(T^{*}\left|T^{n-1}\right|^{2 k} T\right)^{\frac{1}{(n-1) k+1}} & =\left(U^{*}\left|T^{*}\right|\left|T^{n-1}\right|^{2 k}\left|T^{*}\right| U\right)^{\frac{1}{(n-1) k+1}} \\
& =U^{*}\left(\left|T^{*}\right|\left|T^{n-1}\right|^{2 k}\left|T^{*}\right|\right)^{\frac{1}{(n-1) k+1}} U
\end{aligned}
$$

(2.2) holds if and only if

$$
\left(\left|T^{*}\right|\left|T^{n-1}\right|^{2 k}\left|T^{*}\right|\right)^{\frac{1}{(n-1) k+1}} \geq U|T|^{2} U^{*}
$$

if and only if

$$
\left(\left|T^{*}\right|\left|T^{n-1}\right|^{2 k}\left|T^{*}\right|\right)^{\frac{1}{(n-1) k+1}} \geq\left|T^{*}\right|^{2}
$$

Let $A=\left|T^{n-1}\right|^{2 k}$ and $B=\left|T^{*}\right|^{2}$. Then (2.3) is equivalent to the following:

$$
\left(B^{\frac{1}{2}} A B^{\frac{1}{2}}\right)^{\frac{1}{(n-1) k+1}} \geq B \text {. }
$$

By applying Lemma 2.3 to (2.4), for any fixed $\delta \geq-1$,

$$
\begin{aligned}
g(\lambda) & =B^{\frac{-1}{2}}\left(B^{\frac{1}{2}} A^{\lambda} B^{\frac{1}{2}}\right)^{\frac{\delta+1}{(n-1) k \lambda+1}} B^{\frac{-1}{2}} \\
& =\left|T^{*}\right|^{-1}\left(\left|T^{*}\right|\left|T^{n-1}\right|^{2 k \lambda}\left|T^{*}\right|\right)^{\frac{\delta+1}{(n-1) k \lambda+1}}\left|T^{*}\right|^{-1}
\end{aligned}
$$

is increasing for $\lambda \geq 1$ such that $(n-1) k \lambda \geq \delta$. Hence

$$
\begin{aligned}
g(\lambda) & =C^{*(n-1)} g(\lambda) C^{n-1} \\
& =C^{*(n-1)} B^{\frac{-1}{2}}\left(B^{\frac{1}{2}} A^{\lambda} B^{\frac{1}{2}}\right)^{\frac{\delta+1}{(n-1) k \lambda+1}} B^{\frac{-1}{2}} C^{n-1} \\
& =\left(U T^{*} U^{*}\right)^{n-1}\left|T^{*}\right|^{-1}\left(\left|T^{*}\right|\left|T^{n-1}\right|^{2 k \lambda}\left|T^{*}\right|\right)^{\frac{\delta+1}{(n-1) k \lambda+1}}\left|T^{*}\right|^{-1}\left(U T U^{*}\right)^{n-1}
\end{aligned}
$$

is increasing for $\lambda \geq 1$ such that $(n-1) k \lambda \geq \delta$, and we have

$$
\begin{aligned}
g\left(\frac{\ell}{k}\right) & =\left(U T^{*} U^{*}\right)^{n-1}\left|T^{*}\right|^{-1}\left(\left|T^{*}\right|\left|T^{n-1}\right|^{2 \ell}\left|T^{*}\right|\right)^{\frac{\delta+1}{(n-1) \ell+1}}\left|T^{*}\right|^{-1}\left(U T U^{*}\right)^{n-1} \\
& =\left(U T^{*} U^{*}\right)^{n-1}\left|T^{*}\right|^{-1}\left(\left|T^{*}\right|\left|T^{n-1}\right|^{2 \ell}\left|T^{*}\right|\right)^{\frac{\delta+1}{(n-1) \ell+1}}\left|T^{*}\right|^{-1}\left(U T U^{*}\right)^{n-1} \\
& =\left(U T^{*} U^{*}\right)^{n-1}\left|T^{*}\right|^{-1} U\left(\left.T^{*}|| T^{n-1}\right|^{2 \ell} T\right)^{\frac{\delta+1}{(n-1) \ell+1}} U^{*}\left|T^{*}\right|^{-1} T^{n-1} \quad \text { (Since } U \text { is unitary) } \\
& =\left(U T^{*} U^{*}\right)^{n-1} T^{-n^{*}} T^{n-1^{*}}\left(\left.T^{*}|| T^{n-1}\right|^{2 \ell} T\right)^{\frac{\delta+1}{(n-1) \ell+1}} T^{n-1} T^{-n}\left(U T U^{*}\right)^{n-1} \\
& =\left(U T^{*} U^{*}\right)^{n-1} T^{-n^{*}} f_{n, \delta}(\ell) T^{-n}\left(U T U^{*}\right)^{n-1}
\end{aligned}
$$

is increasing for $\ell \geq k$ such that $(n-1) \ell \geq \delta$. Hence $f_{n, \delta}(\ell)$ is increasing for $\ell \geq \max \left\{k, \frac{\delta}{n-1}\right\}$, that is, the proof of Theorem 2.2 is achieved. 
By using Theorem 2.2, we obtain the following results.

Theorem 2.4. Let $T$ be an invertible and quasi-class $A$ operator. Then the following assertions hold;

(a) $T^{*^{n-1}}\left|T^{n}\right|^{\frac{2}{n}} T^{n-1} \geq T^{*^{n-1}}\left(T^{*}\left|T^{n-1}\right|^{\frac{2}{n-1}} T\right)^{\frac{1}{2}} T^{n-1} \geq T^{*}|T|^{2} T$ for $n=$ $2,3, \cdots$

(b) $T^{n *}\left|T^{n+1}\right|^{\frac{2 n}{n+1}} T^{n} \geq T^{* n}\left|T^{n}\right|^{2} T^{n}$ for all positive integer $n$.

(c) $T^{n *}\left|T^{2 n}\right| T^{n} \geq T^{n *}\left|T^{n}\right|^{2} T^{n}$ for all positive integer $n$.

(d) $T^{*}|T|^{2} T \leq T^{*}\left|T^{2}\right| T \leq \cdots \leq T^{* n}\left|T^{n}\right|^{\frac{2}{n}} T^{n}$ for all positive integer $n$.

(e) $T^{*^{-1}}\left|T^{-2}\right| T^{-1} \geq T^{*^{-1}}\left|T^{-1}\right|^{2} T^{-1}$.

Proof. Define $f_{n, \delta}(\ell)$ as (2.1) in Theorem 2.2 .

(a). We will use induction to establish the inequality

$$
\begin{aligned}
T^{*^{n-1}}\left|T^{n}\right|^{\frac{2}{n}} T^{n-1} & \geq T^{*^{n-1}}\left(T^{*}\left|T^{n-1}\right|^{\frac{2}{n-1}} T\right)^{\frac{1}{2}} T^{n-1} \\
& \geq T^{*}|T|^{2} T \text { for } n=2,3, \cdots .
\end{aligned}
$$

In case $n=2$,

$$
T^{*}\left|T^{2}\right| T=T^{*}\left(T^{*}|T|^{2} T\right)^{\frac{1}{2}} T \geq T^{*}|T|^{2} T
$$

hold since $T$ is a quasi-class $A$ operator.

Assume that (2.5) holds for some $n \geq 2$. Then

$T^{*}|T|^{2} T \leq T^{n^{*}}\left(T^{*}|T|^{2} T\right)^{\frac{1}{2}} T^{n} \quad$ (by Inequality (2.5))

$$
\leq T^{n^{*}}\left(T^{*}\left|T^{n}\right|^{\frac{2}{n}} T\right)^{\frac{1}{2}} T^{n} \quad \text { (by Inequality (2.5) and Löwner-Heinz Theorem). }
$$

Then (2.6) and Theorem 2.2 ensure that

$f_{n+1,0}(\ell)=T^{n^{*}}\left(T^{*}\left|T^{n}\right|^{2 \ell} T\right)^{\frac{1}{n \ell+1}} T^{n} \quad$ is increasing for $\ell \geq \max \left\{\frac{1}{n}, 0\right\}=\frac{1}{n}$,

and we have

$$
\begin{aligned}
T^{n^{*}}\left(T^{*}\left|T^{n}\right|^{\frac{2}{n}} T\right)^{\frac{1}{2}} T^{n} & =f_{n+1,0}\left(\frac{1}{n}\right) \\
& \leq f_{n+1,0}(1) \text { by }(2) \\
& =T^{n^{*}}\left(T^{*}\left|T^{n}\right|^{\frac{1}{2}} T\right)^{\frac{1}{n+1}} T^{n} \\
& =T^{n^{*}}\left|T^{n+1}\right|^{\frac{2}{n+1}} T^{n} .
\end{aligned}
$$

Hence (2.6) and (2.8) ensure

$$
T^{n^{*}}\left|T^{n+1}\right|^{\frac{2}{n+1}} T^{n} \geq T^{n^{*}}\left(T^{*}\left|T^{n}\right|^{\frac{2}{n}} T\right)^{\frac{1}{2}} \geq T^{*}|T|^{2} T,
$$


so that (2.5) hold for $n=2,3, \cdots$ by induction, that is, the proof of (a) is achieved.

Proof of (b). We will use induction to establish the inequality

$T^{n *}\left|T^{n+1}\right|^{\frac{2 n}{n+1}} T^{n} \geq T^{* n}\left|T^{n}\right|^{2} T^{n}$ for all positive integer $n$.

In case $n=1, T^{*}\left|T^{2}\right| T \geq T^{*}|T|^{2} T$ holds since $T$ is a quasi-class $A$ operator.

Assume (2.9) holds for some $n$. We remark the following:

since $T^{n^{*}}\left|T^{n+1}\right|^{\frac{2}{n+1}} T^{n} \geq T^{*}|T|^{2} T$ holds by part(a), Theorem 2.2 ensures that

$$
f_{n+2, n}(\ell)=T^{n+1^{*}}\left(T^{*}\left|T^{n+1}\right|^{2 \ell} T\right)^{\frac{n+1}{(n+1) \ell+1}} T^{n+1}
$$

is increasing for $\ell \geq \max \left\{\frac{1}{n+1}, \frac{n}{n+1}\right\}=\frac{n}{n+1}$.

Then we have

$$
\begin{aligned}
T^{n^{*}}\left|T^{n+1}\right|^{2} T^{n} & =T^{n+1^{*}}\left|T^{n}\right|^{2} T^{n+1} \\
& \leq T^{n+1^{*}}\left|T^{n+1}\right|^{\frac{2 n}{n+1}} T^{n+1} \quad \text { (by Inequality (2.9)) } \\
& =f_{n+2, n}\left(\frac{n}{n+1}\right) \quad(\text { by }(2.10)) \\
& \leq f_{n+2, n}(1) \quad(2.11) \\
& =T^{n+1^{*}}\left(T^{*}\left|T^{n+1}\right|^{2} T\right)^{\frac{n+1}{n+2}} T^{n+1} \\
& \left.=T^{n+1^{*}}\left|T^{n+2}\right|\right)^{\frac{2(n+1)}{n+2}} T^{n+1} .
\end{aligned}
$$

Hence (2.9) holds for all positive integer $n$ by induction, that is, the proof of (b) is achieved.

Proof of (c). By part (b) and Löwner-Heinz Theorem, we obtain

$$
\begin{aligned}
T^{n^{*}}\left|T^{n}\right|^{2} T^{n} & \leq T^{n^{*}}\left|T^{n+1}\right| \frac{2 n}{n+1} T^{n}=T^{n^{*}}\left|T^{n+1}\right|^{2 \cdot \frac{n}{n+1}} T^{n} \\
& \leq \cdots \\
& \leq T^{n^{*}}\left|T^{2 n}\right|^{\frac{2(2 n-1}{2 n} \times \frac{n}{2 n-1}} T^{n}=T^{n^{*}}\left|T^{2 n}\right|^{2 \times \frac{n}{2 n}} T^{n} \\
& =T^{n^{*}}\left|T^{2 n}\right| T^{n},
\end{aligned}
$$

so that we have (c).

Proof of (d). Applying Löwner-Heinz Theorem to (b),

$$
T^{n *}\left|T^{n+1}\right|^{\frac{2 n}{n+1}} T^{n} \geq T^{* n}\left|T^{n}\right|^{2} T^{n}
$$

holds for all positive integer $n$. Therefore we obtain

$$
T^{*}|T|^{2} T \leq T^{*}\left|T^{2}\right| T \leq \cdots \leq T^{* n}\left|T^{n}\right|^{\frac{2}{n}} T^{n}
$$


for all positive integer $n$.

Proof of (e). We cite the following obvious result (see [3]): Let $S$ be an invertible operator. Then

$\left(S^{*} S\right)^{\lambda}=S^{*}\left(S S^{*}\right)^{\lambda-1} S$ holds for any real number $\lambda$.

Suppose that $T$ is an invertible quasi-class $A$ operator. Then

$T^{2^{*}} T^{2}=T^{*}|T|^{2} T \leq T^{*}\left|T^{2}\right| T=T^{*}\left(T^{2^{*}} T^{2}\right)^{\frac{1}{2}} T=T^{3^{*}}\left(T^{2} T^{2^{*}}\right)^{\frac{-1}{2}} T^{3}$

holds by (2.12). (2.13) holds if and only if

$$
T^{*^{-1}} T^{-1} \leq\left(T^{*^{-2}} T^{-2}\right)^{\frac{1}{2}}
$$

if and only if

$$
T^{*^{-2}} T^{-2} \leq T^{*^{-1}}\left(T^{*^{-2}} T^{-2}\right)^{\frac{1}{2}} T^{-1}
$$

if and only if

$$
T^{*^{-1}}\left|T^{-1}\right|^{2} T^{-1} \leq T^{*^{-1}}\left|T^{-2}\right| T^{-1},
$$

so that the proof of (e) is complete.

Corollary 2.5. (i) If $T$ is an invertible and quasi-class $A$ operator, then $T^{n}$ is also a quasi-class $A$ operator.

(ii) If $T$ is an invertible and quasi-class $A$ operator, then $T^{-1}$ is also a quasiclass $A$ operator.

Theorem 2.6. Let $T$ be an invertible and quasi-class $A$ operator. Then the following assertions hold;

(a) $T\left|T^{*}\right|^{2} T^{*} \geq T^{n-1}\left(T\left|T^{n-1^{*}}\right|^{\frac{2}{n-1}} T^{*}\right)^{\frac{1}{2}} T^{*^{n-1}} \geq T^{n-1}\left|T^{* n}\right|^{\frac{2}{n}} T^{* n-1}$ for $n=$ $2,3, \cdots$.

(b) $T^{n}\left|T^{n+1^{*}}\right|^{\frac{2 n}{n+1}} T^{n^{*}} \leq T^{n}\left|T^{n^{*}}\right|{ }^{2} T^{n^{*}}$ for all positive integer $n$.

(c) $T^{n}\left|T^{2 n^{*}}\right| T^{n^{*}} \leq T^{n}\left|T^{n^{*}}\right|^{2} T^{n^{*}}$ for all positive integer $n$.

(d) $T\left|T^{*}\right|^{2} T^{*} \geq T\left|T^{2^{*}}\right| T^{*} \geq \cdots \geq T^{n}\left|T^{n^{*}}\right|^{\frac{2}{n}} T^{n^{*}}$ for all positive integer $n$.

Proof. First of all, we remark that

$\left|S^{-1}\right|=\left(S^{*^{-1}} S^{-1}\right)^{\frac{1}{2}}=\left(S S^{*}\right)^{\frac{-1}{2}}=\left|S^{*}\right|^{-1}$ for any invertible operator $S$.

Suppose that $T$ is an invertible and quasi-class $A$ operator. Then $T^{-1}$ is also a quasi-class $A$ operator by part (e) of Theorem 2.4 .

Proof of (a). Since $T^{-1}$ a quasi-class $A$ operator, applying part (a) of Theorem 2.4 , we have

$T^{*^{-n+1}}\left|T^{-n}\right|^{\frac{2}{n}} T^{-n+1} \geq T^{*^{-n+1}}\left(T^{-1^{*}}\left|T^{-n+1}\right| \frac{2}{n-1} T^{-1}\right)^{\frac{1}{2}} T^{-n+1} \geq T^{-1^{*}}\left|T^{-1}\right|^{2} T^{-1}$. 
By (2.15), (2.16) hold if and only if $T^{*^{-n+1}}\left|T^{n^{*}}\right|^{\frac{-2}{n}} T^{-n+1} \geq T^{*^{-n+1}}\left(T^{-1^{*}}\left|T^{n-1^{*}}\right|^{\frac{-2}{n-1}} T^{-1}\right)^{\frac{1}{2}} T^{-n+1} \geq T^{-1^{*}}\left|T^{*}\right|^{-2} T^{-1}$.

if and only if

$$
T^{n-1}\left|T^{n^{*}}\right|^{\frac{2}{n}} T^{n-1^{*}} \leq T^{n-1}\left(T\left|T^{n-1^{*}}\right|^{\frac{2}{n-1}} T^{*}\right)^{\frac{1}{2}} T^{n-1^{*}} \leq T\left|T^{*}\right|^{2} T^{*} .
$$

Proof of (b). Since $T^{-1}$ a quasi-class $A$ operator, applying part (b) of Theorem 2.4, we have

$$
T^{(-n) *}\left|T^{-(n+1)}\right|^{\frac{2 n}{n+1}} T^{-n} \geq T^{(-n)^{*}}\left|T^{-n}\right|^{2} T^{-n} .
$$

By (2.15), (2.17) hold if and only if

$$
T^{(-n) *}\left|T^{(n+1)^{*}}\right|^{\frac{-2 n}{n+1}} T^{-n} \geq T^{(-n)^{*}}\left|T^{n^{*}}\right|^{-2} T^{-n} .
$$

if and only if

$$
T^{n}\left|T^{(n+1)^{*}}\right|^{\frac{2 n}{n+1}} T^{n^{*}} \leq T^{n}\left|T^{n^{*}}\right|^{2} T^{n^{*}}
$$

Proof of (c). Since $T^{-1}$ a quasi-class $A$ operator, applying part (c) of Theorem 2.4 , we have

$$
T^{(-n)^{*}}\left|T^{-2 n}\right| T^{-n} \geq T^{(-n)^{*}}\left|T^{-n}\right|^{2} T^{-n} .
$$

By (2.15), (2.18) hold if and only if

$$
T^{(-n)^{*}}\left|T^{(2 n)^{*}}\right|^{-1} T^{-n} \geq T^{(-n)^{*}}\left|T^{n^{*}}\right|^{-2} T^{-n} .
$$

if and only if

$$
T^{n}\left|T^{(2 n)^{*}}\right| T^{n^{*}} \leq T^{n}\left|T^{n^{*}}\right|^{2} T^{n^{*}} .
$$

Proof of (d). Since $T^{-1}$ a quasi-class $A$ operator, applying part (d) of Theorem 2.4, we have

$T^{*^{-1}}\left|T^{-1}\right|^{2} T^{-1} \leq T^{-1 *}\left|T^{-2}\right| T^{-1} \leq \cdots \leq T^{(-n)^{*}}\left|T^{-n}\right|^{\frac{2}{n}} T^{-n}$.

By (2.15), (2.19) hold if and only if

$$
T^{*^{-1}}\left|T^{*}\right|^{-2} T^{-1} \leq T^{*^{-1}}\left|T^{2^{*}}\right|^{-1} T^{-1} \leq \cdots \leq T^{(-n)^{*}}\left|T^{n^{*}}\right|^{\frac{-2}{n}} T^{-n} .
$$

if and only if

$$
T\left|T^{*}\right|^{2} T^{*} \geq T\left|T^{2^{*}}\right| T^{*} \geq \cdots \geq T^{n}\left|T^{n^{*}}\right|^{\frac{2}{n}} T^{n^{*}} .
$$

Hence the proof of the theorem is achieved. 
Hölder-McCarthy Inequality. Let $T$ be a positive operator. Then the following inequalities hold for all $x \in \mathcal{H}$ :

(i) $\left\langle T^{r} x, x\right\rangle \leq\langle T x, x\rangle^{r}\|x\|^{2(1-r)}$ for $0<r \leq 1$.

(ii) $\left\langle T^{r} x, x\right\rangle \geq\langle T x, x\rangle^{r}\|x\|^{2(1-r)}$ for $r \geq 1$.

Theorem 2.7. Let $T$ be a quasi-class $A$. Then the following assertions hold.

(i) $\left\|T^{k+1} x\right\|^{2} \leq\left\|T^{k} x\right\|\left\|T^{k+2} x\right\|$ for all unit vectors $x \in \mathcal{H}$ and all positive integer $k$.

(ii) $\left\|T^{k+1}\right\|^{k+1} \leq r\left(T^{k+1}\right)\left\|T^{k}\right\|^{k+1}$ for all positive integer $k$, where $r\left(T^{k}\right)$ denote the spectral radius of $T^{k}$.

Proof. (i) Suppose that $T$ is a quasi-class $A$. Then for every unit vector $x \in \mathcal{H}$, we have

$$
\begin{aligned}
\left\|T^{k+1} x\right\|^{2} & =\left\langle T^{* k}|T|^{2} T^{k} x, x\right\rangle \\
& \leq\left\langle T^{* k}\left|T^{2}\right| T^{k} x, x\right\rangle \\
& \leq\left\langle\left(T^{* 2} T^{2}\right)^{1 / 2} T^{k} x, T^{k} x\right\rangle \\
& \leq\left\langle\left(T^{* 2} T^{2}\right) T^{k} x, T^{k} x\right\rangle^{1 / 2}\left\|T^{k} x\right\| \\
& \leq\left\|T^{k+2} x\right\|\left\|T^{k} x\right\| .
\end{aligned}
$$

(ii) If $T^{k}=0$ for some $k>1$, then $r\left(T^{k}\right)=0$. Hence (ii) is obvious. Hence we may assume $T^{k} \neq 0$ for all $k \geq 1$. Then

$$
\frac{\left\|T^{k+1}\right\|}{\left\|T^{k}\right\|} \leq \frac{\left\|T^{k+2}\right\|}{\left\|T^{k+1}\right\|} \leq \cdots \leq \frac{\left\|T^{m(k+1)}\right\|}{\left\|T^{m(k+1)-1}\right\|}
$$

by (i), and we have

$$
\left(\frac{\left\|T^{k+1}\right\|}{\left\|T^{k}\right\|}\right)^{m(k+1)-k} \leq \frac{\left\|T^{k+1}\right\|}{\left\|T^{k}\right\|} \times \cdots \times \frac{\left\|T^{m(k+1)}\right\|}{\left\|T^{m(k+1)-1}\right\|}=\frac{\left\|T^{m(k+1)}\right\|}{\left\|T^{k}\right\|} .
$$

Hence

$$
\left(\frac{\left\|T^{k+1}\right\|}{\left\|T^{k}\right\|}\right)^{(k+1)-\frac{k}{m}} \leq \frac{\left\|T^{m(k+1)}\right\|^{\frac{1}{m}}}{\left\|T^{k}\right\|^{\frac{1}{m}}},
$$

letting $m \rightarrow \infty$, we have

$$
\left\|T^{k+1}\right\|^{k+1} \leq r\left(T^{k+1}\right)\left\|T^{k}\right\|^{k+1} .
$$




\section{References}

[1] A. Aluthge, D. Wang, An operator inequality which implies paranormality. Math. Ineq. Appl. 2 (1) (1999), 113-119.

[2] A. Aluthge, D. Wang, w-hyponormal operators. Integral Equation Operator Theory, 36 (2000), 1-10.

[3] M. Chō, T. Yamazaki, An operator transform from class $A$ to the class of hyponormal operators and its application. Integral Equation operator Theory $5 \mathbf{3}$ (4) (2005), 497-508.

[4] B. P. Duggal, I. H. Jeon and I. H. Kim , On Weyl's theorem for quasi-class $A$ operators. J. Korean Math. Soc., 43 (4) (2006), 899-909.

[5] T. Furuta, M. Ito and T. Yamazaki, A subclass of paranormal operators including class of $l o g$-hyponormal and several related classes. Sci. math. 1 (1998), 389-403.

[6] M. Ito, Several Properties On Class $A$ including $p-$ hyponormal and log-hyponormal operators. Math. Ineq. Appl. 2 (4)(1999), 569-578.

[7] I. H. Jeon, I. H. Kim, On operators satisfying $T^{*}\left|T^{2}\right| T \geq T^{*}|T|^{2} T^{*}$. Linear Alg. Appl. 418 (2006), 854-862.

[8] I. H. Jeon, J.I. Lee and A. Uchiyama, On p-quasihyponormal operators and quasisimilarity. Math. Ineq. App. 6 (2) (2003), 309-315.

[9] I.H. Kim, On (p,k)-quasihyponormal operators. Math. Ineq. Appl. 4 (2004), 169-178.

[10] A. Uchiyama, Weyl's theorem for Class $A$ operators. Math. Ineq. App. 4 (1) (2001), 143-150.

M.H.M.Rashid

Department of Mathematics\& Statistics

Faculty of Science P.O.Box(7)

Mu'tah University

Al-Karak-Jordan 